\title{
The influence of acute physical effort on liver stiffness estimation using Virtual Touch Quantification (VTQ). Preliminary results.
}

\author{
Mariana M Gersak ${ }^{1}$, Erich Sorantin², Jana Windhaber ${ }^{3}$, Sorin M Dudea ${ }^{1}$, Michael Riccabona ${ }^{2}$ \\ ${ }^{1}$ Department of Radiology, Cluj County Emergency Hospital, University of Medicine and Pharmacy "Iuliu Hatie- \\ ganu", Cluj-Napoca, Romania, ${ }^{2}$ Department of Radiology, Division of Pediatric Radiology, Medical University of \\ Graz, Austria, ${ }^{3}$ Department of Pediatric and Adolescent Surgery, Medical University of Graz, Austria
}

\begin{abstract}
Aim: Food intake and acutely decompensated cardiac failure may impair liver stiffness estimations, mainly because these methods are influenced by liver blood flow variation encountered in these conditions. It is well known that also during physical effort liver blood flow changes, due to the redistribution of blood flow mainly to the skeletal muscles. The aim of this study is to assess the change of liver shear- wave velocities immediately after cessation of an acute physical effort. A secondary aim was to find the proper timing for accurate liver stiffness estimation after physical exhaustion. Material and methods: Liver shear-wave velocities were measured using virtual touch quantification (VTQ) in seven apparently healthy volunteers, in fasting conditions. All subjects underwent a complete abdominal ultrasound study, including the liver VTQ. Then, all subjects performed a spiroergometry and thereafter, another series of three liver shear-wave velocity measurements were performed: immediately after spiroergometry, five min, and ten min after spiroergometry, respectively. Results: Before spiroergoemetry, the mean liver shear-wave velocity was $1.05 \pm 0.12 \mathrm{~m} / \mathrm{sec}$. Immediately after spiroergometry, mean liver shear-wave velocities increased significantly, measuring $1.34 \pm 0.16 \mathrm{~m} / \mathrm{sec}(\mathrm{p}<0.01)$. The mean liver shear-wave velocities at five and ten minutes after exhausting were $1.23 \pm 0.14 \mathrm{~m} / \mathrm{sec}$ and $1.05 \pm 0.11 \mathrm{~m} / \mathrm{sec}$ respectively. Conclusions: Liver stiffness estimation using VTQ was influenced by acute physical exercise in our study group. Despite the small number of subjects, the preliminary results show that if patients had done physical effort before VTQ elastography, they should rest at least ten minutes before reliable liver stiffness estimations can be performed.
\end{abstract}

Keywords: liver, stiffness, VTQ, elastography, physical exercise.

\section{Introduction}

Estimation of the liver stiffness can be achieved by using multiple types of liver elastography such as transient elastography (TE), strain elastography (SE), two-dimensional sonographic shear wave elastography (2D-SWE), and also MR elastography. Virtual Touch Quantification (VTQ) elastography uses focused ultrasound (US) pulses which are sent to the tissue using a conventional US transducer and dedicated equipment /

Received 24.12.2015 Accepted 17.02.2016

Med Ultrason

2016, Vol. 18, No 2, 151-156

Corresponding author: Mariana M. Gersak

3-5 Clinicilor street,

400006, Cluj-Napoca, Romania

Phone: +40 741922642

E-mail: mariana.gersak@umfcluj.ro software. The tissue responds to these pulses with different amount of displacement creating shear waves of variable velocities [1]. The stiffer the tissue is, the higher is the shear wave velocity - thus these shear wave velocities can be used to estimate stiffness of the respective tissue; the velocity measurements are usually given in (c) $\mathrm{m} / \mathrm{sec}$ and can be transferred by simple equations in Pascals $[2,3]$. As tissues have different components, ultra-structural compositions and densities, normal shear wave velocity cutoff values also vary [4]. For example, in kidneys, shear wave velocities are influenced mainly by external pressure, tissue vascularization, intravascular pressure, and tissue anisotropy [1,5]. Thus, increased shear wave velocities were described without a necessarily stiffer kidney parenchyma, just due to different tissue compartments, anisotropy, the high vascularization and elevated urinary collecting system pressure [5]. Some of 
these parameters are responsible also for increased liver shear wave velocities in different pathologies, actually without a stiffer liver parenchyma itself. Examples for this phenomenon are cholestasis, increased level of liver enzymes, acutely decompensated cardiac failure; also food intake may impair liver elasticity values derived from US elastography [6-11]. There are studies describing that liver stiffness measurements increase immediately after food intake, most probably due to a markedly increased blood flow in the portal vein [12]. In acutely decompensated heart failure, there is an increased venous pressure causing stasis in the inferior vena cava, influencing blood flow in the intrahepatic liver veins which is also transmitted into the liver parenchyma and therefore, liver stiffness appears increased [6]. Another cause of blood flow redistribution is during physical effort. There are many studies describing some sort of physiological gastrointestinal ischemia during physical exercise, as more than $50 \%$ of the lower abdominal arterial blood flow is redistributed to the muscles [13-16]. This assumption suggests that during physical exercise liver stiffness measurements will be impacted too and probably should be lower than baseline values. To our knowledge, there are no studies which describe how physical exercise influences liver stiffness estimations. Moreover, it would be of great clinical importance to know, what happens with liver stiffness estimations after physical exercise, especially, to know the appropriate timing for accurate liver stiffness estimation tests.

The purpose of this prospective study was to evaluate if liver stiffness measurements are influenced by physical exercise in apparently healthy subjects and, if so, also to determine when liver stiffness measurements return to baseline values.

\section{Materials and methods}

\section{Subjects}

Seven healthy volunteers $(32.14 \pm 4.52$ years, 6 females and 1 male) were included in this pilot study. All volunteers were medical staff, whose annual blood samples were within normal limits, with no history of liver or heart disease. All volunteers were physicians. They agreed to participate in this pilot study without any remuneration; according to national regulations and local institutional policy, the Ethical Board approval for this study was waived.

\section{Study design.}

The study protocol was as follows:

Step 1: a baseline abdominal US examination including VTQ elastography was performed using an Acuson S3000 (Siemens Inc., Forchheim, Germany) device with a 6C1HD abdominal curved array transducer by a radiologist with four years of experience in liver elastography. VTQ liver stiffness estimation was always performed in fasting conditions, following international guidelines $[17,18]$.

Step 2 consisted of a spiroergometry which was performed in a stepwise fashion [19]. Exercise stress testing was performed as an incremental exercise on a cycle ergometer (Excalibur Sport, company LODE). The workload was increased every minute until exhaustion [19]. ECG and oxygen consumption were continuously recorded, blood pressure and lactate were measured every two minutes. Maximal heart rate, maximal lactate, and respiratory exchange ratio were documented as a sign of exhaustion.

Step 3 was represented by three consecutive series of liver stiffness estimation: immediately after spiroergometry as well as five minutes and ten minutes after spiroergometry respectively.

For liver stiffness estimation, the volunteers were lying in supine position, with the right hand placed above the head. The transducer was placed perpendicular to the abdominal wall avoiding intrahepatic vessels, ligaments and the gallbladder. Each time, liver elasticity was assessed in the same intercostal space in the mid-axillary line in the right liver lobe. The region of interest (ROI) was placed at least $1 \mathrm{~cm}$ under the liver capsule, where the liver parenchyma was at least $5 \mathrm{~cm}$ thick. The depth and position of the ROI was established for each subject during the first series of liver VTQ elastography individually and then was kept constant for all measurements. When the ROI was placed in the proper place, the subjects stopped their breath for a few seconds, till the shear wave velocity was acquired. The median of ten correctly performed measurements was used to estimate the liver stiffness value for every series of measurement. The individual increase of liver shear wave velocities was calculated immediately after exercise using the following formula: $\%=$ (shear wave velocity immediately after exercise - shear wave velocity before exercise)/ shear wave velocity before exercise x 100).

As additional criteria for accurate liver stiffness estimation, IQR (interquartile range) and SR (success rate) were calculated, according to EFSUMB guidelines $[17,18]$.

\section{Statistical analysis}

For statistics, Microsoft Excel and IBM ${ }^{\circledR}$ SPSS Statistics (v.19.0 Armonk, NY) software packages were used, both for data representation and for statistical analysis. Data distribution was tested with the Shapiro-Wilk test and then, the MANOVA test for multiple measurements and the t-test for paired samples were 
applied. The paired t-tests outcomes were checked by the bootstrap method, for a 100 patients, with similar characteristics.

\section{Results}

One subject stopped the exercise before exhaustion due to muscle pain. Although an elastography was performed immediately after she had stopped the exercise, she was excluded from further statistical analysis. Thus, finally, a total of six healthy subjects were available for analysis ( 5 female, 1 male) with a mean age of $32 \pm 4.94$ years and a body mass index (BMI) of $21.83 \pm 4.29 \mathrm{~kg} /$ $\mathrm{m}^{2}$. Mean liver shear wave velocities were $1.05 \pm 0.12 \mathrm{~m} /$ sec before exercise, $1.34 \pm 0.16 \mathrm{~m} / \mathrm{sec}$ immediately after exercise, $1.23 \pm 0.14 \mathrm{~m} / \mathrm{sec}$ five minutes after exercise, and $1.05 \pm 0.11 \mathrm{~m} / \mathrm{sec}$ ten minutes after exercise. For all series of measurements, IQR was below $30 \%$ and SR above $60 \%$.

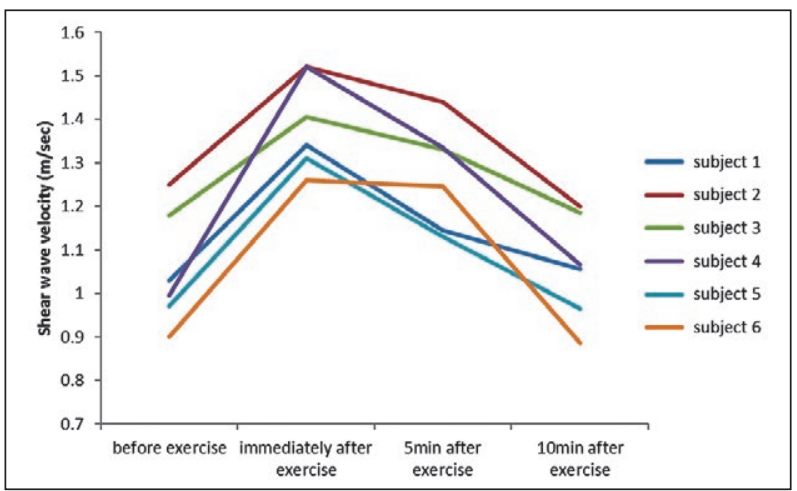

Fig 1. Individual liver shear wave velocity values over time $(\mathrm{m} / \mathrm{sec})$. Data before, immediately after, five minutes after, and ten minutes after exercise. Immediately after exercise, liver stiffness values increased abruptly, and then slowly returned to baseline values within ten minutes.

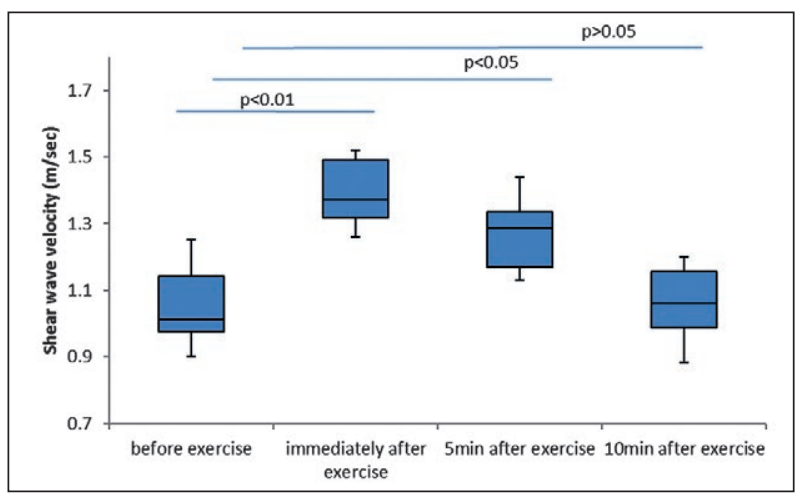

Fig 2. Comparison of shear wave velocities. The individual median values at each point of time during the measurement series are given in range (blue boxes) and standard deviation (whisker).
The single subject who had stopped the exercise complaining of muscle pain only had a $1.43 \%$ increase in liver shear wave velocity immediately after she stopped the exercise. The other six subjects who successfully finished the exercise until exhaustion had highly increased in liver shear wave velocities $(19.07 \%, 21.60 \%, 30.10 \%$, $35.05 \%, 40.00 \%$, and $52.76 \%$ ); development of the individual liver shear wave velocity values over time are shown in figure 1. Using the MANOVA test for multiple measurements, there were significant differences between the four series of liver stiffness evaluation (before exercise, immediately after exercise, five min and ten minutes after effort), $\mathrm{p}<0.01$. Then, as a post-hoc data analysis, we applied paired t-tests to find where the differences between these measurements were. Immediately after spiroergometry liver stiffness values were increased compared with baseline values $(p<0.01)$. There were also significant differences between the values immediately after physical effort and five minutes after the exercise $(\mathrm{p}<0.01)$. No significant differences were identified between baseline values and the last measurement, at ten minutes after exercise ( $p>0.05$ ) (fig 2). Using the bootstrap method, a sample of 100 patients with similar characteristics was simulated. This method reinforced the outcomes of the paired t-tests applied for our study sample, showing the p-values in the same fashion as the study sample.

\section{Discussions}

Many parameters may cause higher intrahepatic tissue shear wave velocities (e.g. fibrosis, acutely decompensated cardiac failure, increased level of liver enzymes, food intake, and cholestasis); in such conditions there is also an increased intracellular hepatic pressure or increased liver, mainly portal venous, blood flow - both also influencing liver stiffness. Most probably, those latter phenomena are explained by the fact that the liver capsule is relatively inextensible and does not allow large expansion in sudden liver volume variations. There are a few physiological and pathological conditions that are affected by these variations and may - when using tissues sound propagation velocity depending methods for liver stiffness estimation (such as US elastography methods) be influenced by these situations such as increased postprandial liver blood supply immediately after food intake $[9,10,12]$ or with acutely decompensated heart failure $[6,7]$ and with extra-hepatic cholestasis $[8,9]$.

Another, yet undescribed potential liver stiffness estimation variation may occur in comparable physiological conditions which are associated with the redistribution of arterial blood flow such as during physical exercise. It 
is well known that during physical effort heart rate and blood pressure are increasing and blood flow is redistributed mostly to the skeletal muscles. During physical exercise, splanchnic blood flow decreases to more than $50 \%$ of baseline values due to sympathetic activity [20]. In healthy subjects, heart rate returns to baseline values in around 1-14 min after the end of the physical exercise and mean blood pressure returns to baseline values in around 3-8 min, depending on exercise level intensities [16]. Also, the decreased lower abdominal arterial blood flow returns to baseline values in around 2-6 min after the end of physical exercise in healthy subjects [16]. Portal venous flow decreases during exercise, down to $80 \%$ of baseline values $[21,22]$. But the literature also describes a "hepatic arterial buffer response" phenomenon, i.e., the hepatic artery dilates to compensate for the decreased portal venous flow [23-25]. This response is due to adenosine accumulation and may compensate up to $25-60 \%$ of the decreased portal venous flow $[24,26]$. However, some authors found that after physical exercise, the portal venous flow velocity remained relatively unchanged and the decreased portal blood flow volume was then explained by a decreased caliber of the portal vein [27]. When analyzing the liver blood flow velocities after exercise, hepatic veins are also important. There are reports on increased blood flow velocities in hepatic veins after exercise, with values up to $148 \%$ in the systolic phase of the three-phasic undulating liver vein flow profile, up to $139 \%$ for diastolic phase, and up to $372 \%$ during atrial contraction - with a consecutive backward flow into the hepatic veins [27]. Thus, immediately after exercise, there is a considerable regurgitation flow in hepatic veins, probably related to atrial contractions. Finally, motion transmitted from heart beat and aortic pulsations may cause artificially increased elastography results - this however has been reported only for the left liver lobe [28]; our measurements were taken in the right liver lobe. It nevertheless may be another reason explaining our results: if one considers the increased heart frequency, stronger heart beats and aortic pulsations as obviously present in the state of heavy physical effort it might be hypothesized that these motions are event transmitted to the right liver lobe and thus this may be one of the factors why US elastography measurements immediately after physical exercise are unreliable.

Our results show that liver shear wave velocities used for estimating liver stiffness increase immediately after physical exercise and return to normal after some rest, in a similar fashion as the other factors that indicate the state of the circulation regulation. The reason for the obvious and significant increased shear wave velocities, however, remains unclear. Most probably, the markedly increased regurgitation flow velocities in hepatic veins during atrial contraction might explain the impact on liver shear wave velocities, with an increase up to $52 \%$. It is also possible that the hepatic arterial buffer response might have an additional contribution to an increased intrahepatic pressure and thus might contribute to the elevated "stiffness" values found in this study; but probably also more complex mechanisms might be responsible for this marked increased shear wave velocity after exhaustion which are not yet completely understood. Potentially also some underlying physical phenomena intrinsic to the measurement method may also contribute to these findings, such as US pulse wave and shear wave propagation or depiction.

Nevertheless - in our series liver shear wave velocities returned to the baseline values by ten minutes after cessation of the physical effort, when heart rate, blood pressure and lower abdominal arterial blood flow return to baseline values in healthy subjects [16]. This observation, however, confirms that only physical exercise is responsible for inducing the observed shear wave velocity changes.

Many studies have shown that most of the factors which impair an accurate TE liver stiffness estimation were also shown to impair VTQ elastography estimations (for example, food intake, increased aminotransferase levels, cholestasis, acutely decompensated heart failure) [6-11,29,30]. Thus, it is reasonable to expect that physical exercise may also impair TE or even 2D-SWE, as both are based on shear wave measurements, similar to VTQ- although to date studies on these factors have not been published.

Why are these observations clinically important? Knowledge of the observed VTQ value variation after physical exercise can have a considerable impact on patient management: for example in children, who have been playing or running before they come in for the US examination with VTQ elastography; these impaired values may incorrectly suggest a non-existing liver disease. The same applies to adult patients, who are in a hurry to arrive in time for the liver stiffness estimation appointment - biased values could be the consequence with inherent negative impact on their clinical management.

There are limitations of this pilot study too that need to be considered and discussed. (1) The most important limitation of the present study is the small sample size. But considering the observed consistent and large liver shear wave velocity change / increase after physical exercise that was statistically significant even in such a small sample size implies that this phenomenon needs to be considered to avoid bias during such liver stiffness tests. Also, the bootstrap method for a larger, similar sample reinforced the outcomes of our study sample, making the observed differences more reliable. (2) The study design did not include a control group, considering the publica- 
tion who showed that liver stiffness estimation by VTQ did not vary significantly in time, in the control group [10] and also the good intraobserver agreement with an intraclass correlation coefficient (ICC) is up to 0.91[31]. However, we acknowledge that the absence of the control group is another limitation of the study. (3) No blood tests for liver function were performed; the subjects were considered healthy only based on their history without any liver, gastrointestinal, or cardiac disease; their abdominal (and liver) US was normal, and the parameters measured during spiroergometry were within normal limits. Furthermore, baseline liver stiffness values within the normal range in the basic examination also are suggestive of healthy subjects [32]. (4) Finally, there were some technical limitations during this study - consecutive measurements of blood pressure, heart rate or liver hemodynamics after spiroergometry could not be performed, mainly because it would have taken too long to perform the liver elastography measurements without delay. But the dynamics and variation of these parameters in relation to physical exercise are known in healthy subjects and for liver hemodynamics from several larger studies; furthermore, the assessment of these parameters was not the main objective of this study.

\section{Conclusions}

Liver stiffness estimation based on shear wave velocity measurements using the VTQ method had an important transitory variance after physical exercise in our study group. Increased values up to $52 \%$ above baseline liver stiffness values immediately have been observed after the end of acute exhausting physical exercise - this might be an important phenomenon that needs to be acknowledged, respected and considered to avoid false interpretation of increased liver stiffness estimates when performing shear wave velocity based VTQ elastography shortly after physical exercise. To avoid misleading increased values, we recommend that patients should avoid physical exercise before liver stiffness estimation or they should rest at least for ten minutes after the physical exercise before the elastography test. However, although this study shows that liver shear wave velocities have an obvious tendency to increase after physical exercise, the small number in this pilot study implies that this needs to be confirmed in a larger sample and cross-checked with other elastography methods.

Acknowledgments: To all the volunteers included, for their willingness to participate in this study without any reimbursement or other benefit for their contribution.

Conflict of interest: none

\section{References:}

1. Stenzel M, Mentzel HJ. Ultrasound elastography and contrast-enhanced ultrasound in infants, children and adolescents. Eur J Radiol 2014; 83: 1560-1569.

2. Nightingale KR, Palmeri ML, Nightingale RW, Trahey GE. On the feasibility of remote palpation using acoustic radiation force. J Acoust Soc Am 2001; 110: 625-634.

3. Nightingale K, McAleavey S, Trahey G. Shear-wave generation using acoustic radiation force: in vivo and ex vivo results. Ultrasound Med Biol 2003; 29: 1715-1723.

4. Jiang Y, Li GY, Qian LX, et al. Characterization of the nonlinear elastic properties of soft tissues using the supersonic shear imaging (SSI) technique: inverse method, ex vivo and in vivo experiments. Med Image Anal 2015; 20: 97-111.

5. Gennisson JL, Grenier N, Combe C, Tanter M. Supersonic shear wave elastography of in vivo pig kidney: influence of blood pressure, urinary pressure and tissue anisotropy. Ultrasound Med Biol 2012; 38: 1559-1567.

6. Colli A, Pozzoni P, Berzuini A, et al. Decompensated chronic heart failure: increased liver stiffness measured by means of transient elastography. Radiology 2010; 257: 872-878.

7. Goertz RS, Egger C, Neurath MF, Strobel D. Impact of food intake, ultrasound transducer, breathing maneuvers and body position on acoustic radiation force impulse (ARFI) elastometry of the liver. Ultraschall Med 2012; 33: 380-385.

8. Millonig G, Reimann FM, Friedrich S, et al. Extrahepatic cholestasis increases liver stiffness (FibroScan) irrespective of fibrosis. Hepatology 2008; 48: 1718-1723.

9. Pfeifer L, Strobel D, Neurath MF, Wildner D. Liver stiffness assessed by acoustic radiation force impulse (ARFI) technology is considerably increased in patients with cholestasis. Ultraschall Med 2014; 35: 364-367.

10. Popescu A, Bota S, Sporea I, et al. The influence of food intake on liver stiffness values assessed by acoustic radiation force impulse elastography-preliminary results. Ultrasound Med Biol 2013; 39: 579-584.

11. Sagir A, Erhardt A, Schmitt M, Häussinger D. Transient elastography is unreliable for detection of cirrhosis in patients with acute liver damage. Hepatology 2008; 47: 592-595.

12. Dauzat M, Lafortune M, Patriquin H, Pomier-Layrargues G. Meal induced changes in hepatic and splanchnic circulation: a noninvasive Doppler study in normal humans. Eur J Appl Physiol Occup Physiol 1994; 68: 373-380.

13. Qamar MI, Read AE. Effects of exercise on mesenteric blood flow in man. Gut 1987 ; 28: 583-587.

14. Osada T, Katsumura T, Hamaoka T, et al. Reduced blood flow in abdominal viscera measured by Doppler ultrasound during one-legged knee extension. J Appl Physiol 1999; 86: 709-719.

15. Rowell LB, Blackmon JR, Bruce RA. Indocyanine Green Clearance and Estimated Hepatic Blood Flow during Mild to Maximal Exercise in Upright Man. J Clin Invest 1964; 43: $1677-1690$.

16. Osada $T$, Iwane H, Katsumura $T$, et al. Relationship between reduced lower abdominal blood flows and heart rate in recovery following cycling exercise: Reduced blood flow in lower abdomen after exercise. Acta Physiol 2012; 204: 344-353. 
17. Cosgrove D, Piscaglia F, Bamber J, et al. EFSUMB guidelines and recommendations on the clinical use of ultrasound elastography.part 2: clinical applications. Ultraschall Med 2013; 34: 238-253

18. Sporea I, Bota S, Săftoiu A, et al. Romanian national guidelines and practical recommendations on liver elastography. Med Ultrason 2014; 16: 123-138.

19. Wonisch M, Berent R, Klicpera M, et al. Praxisleitlinien ergometrie. J Kardiol 2008; 15(Suppl A): 3-17.

20. Thijssen DH, Steendijk S, Hopman MT. Blood redistribution during exercise in subjects with spinal cord injury and controls. Med Sci Sports Exerc 2009; 41: 1249-1254.

21. Rehrer NJ, Smets A, Reynaert H, Goes E, De Meirleir K. Effect of exercise on portal vein blood flow in man. Med Sci Sports Exerc 2001; 33: 1533-1537.

22. Rehrer NJ, Goes E, DuGardeyn C, Reynaert H, DeMeirleir $\mathrm{K}$. Effect of carbohydrate on portal vein blood flow during exercise. Int J Sports Med 2005; 26: 171-176.

23. Jakab F, Ráth Z, Schmal F, Nagy P, Faller J. The interaction between hepatic arterial and portal venous blood flows; simultaneous measurement by transit time ultrasonic volume flowmetry. Hepatogastroenterology 1995; 42: 18-21.

24. Lautt WW, Legare DJ, d'Almeida MS. Adenosine as putative regulator of hepatic arterial flow (the buffer response). Am J Physiol 1985; 248: H331-H338.

25. Lautt WW, Legare DJ, Ezzat WR. Quantitation of the hepatic arterial buffer response to graded changes in portal blood flow. Gastroenterology 1990; 98: 1024-1028.
26. Lautt WW. Relationship between hepatic blood flow and overall metabolism: the hepatic arterial buffer response. Fed Proc 1983; 42: 1662-1666.

27. Teichgräber UK, Gebel M, Benter T, Manns MP. Effect of respiration, exercise, and food intake on hepatic vein circulation. J Ultrasound Med 1997; 16: 549-554.

28. Toshima T, Shirabe K, Takeishi K, et al. New method for assessing liver fibrosis based on acoustic radiation force impulse: a special reference to the difference between right and left liver. J Gastroenterol 2011; 46: 705-711.

29. Arena U, Lupsor Platon M, Stasi C, et al. Liver stiffness is influenced by a standardized meal in patients with chronic hepatitis $\mathrm{C}$ virus at different stages of fibrotic evolution. Hepatology 2013; 58: 65-72.

30. Bota S, Sporea I, Peck-Radosavljevic M, et al. The influence of aminotransferase levels on liver stiffness assessed by Acoustic Radiation Force Impulse Elastography: a retrospective multicentre study. Dig Liver Dis 2013; 45: 762768.

31. Bota S, Sporea I, Sirli R, Popescu A, Danila M, Costachescu D. Intra- and interoperator reproducibility of acoustic radiation force impulse (ARFI) elastography--preliminary results. Ultrasound Med Biol 2012; 38: 1103-1108.

32. Liao LY, Kuo KL, Chiang HS, Lin CZ, Lin YP, Lin CL. Acoustic radiation force impulse elastography of the liver in healthy patients: test location, reference range and influence of gender and body mass index. Ultrasound Med Biol 2015; 41: 698-704. 